Revue de droit comparé du travail et de la sécurité sociale

\title{
2| 2019
}

L'ubérisation du travail

\section{Uber et la réglementation canadienne : état de la jurisprudence}

\section{Urwana Coiquaud}

\section{(2) OpenEdition}

1 Journals

Édition électronique

URL : https://journals.openedition.org/rdctss/1560

DOI : $10.4000 /$ rdctss. 1560

ISSN : 2262-9815

Éditeur

Centre de droit comparé du travail et de la sécurité sociale

\section{Édition imprimée}

Date de publication : 1 juin 2019

Pagination : 82-89

ISSN : 2117-4350

\section{Référence électronique}

Urwana Coiquaud, «Uber et la réglementation canadienne : état de la jurisprudence », Revue de droit comparé du travail et de la sécurité sociale [En ligne], 2 | 2019, mis en ligne le 01 novembre 2021, consulté le 11 novembre 2021. URL : http://journals.openedition.org/rdctss/1560 ; DOI : https:// doi.org/10.4000/rdctss. 1560

\section{(c) (†) $९$}

Revue de droit comparé du travail et de la sécurité sociale est mise à disposition selon les termes de la Licence Creative Commons Attribution - Pas d'Utilisation Commerciale - Pas de Modification 4.0 International. 


\section{UBER ET LA RÉGLEMENTATION CANADIENNE : ÉTAT DE LA JURISPRUDENCE}
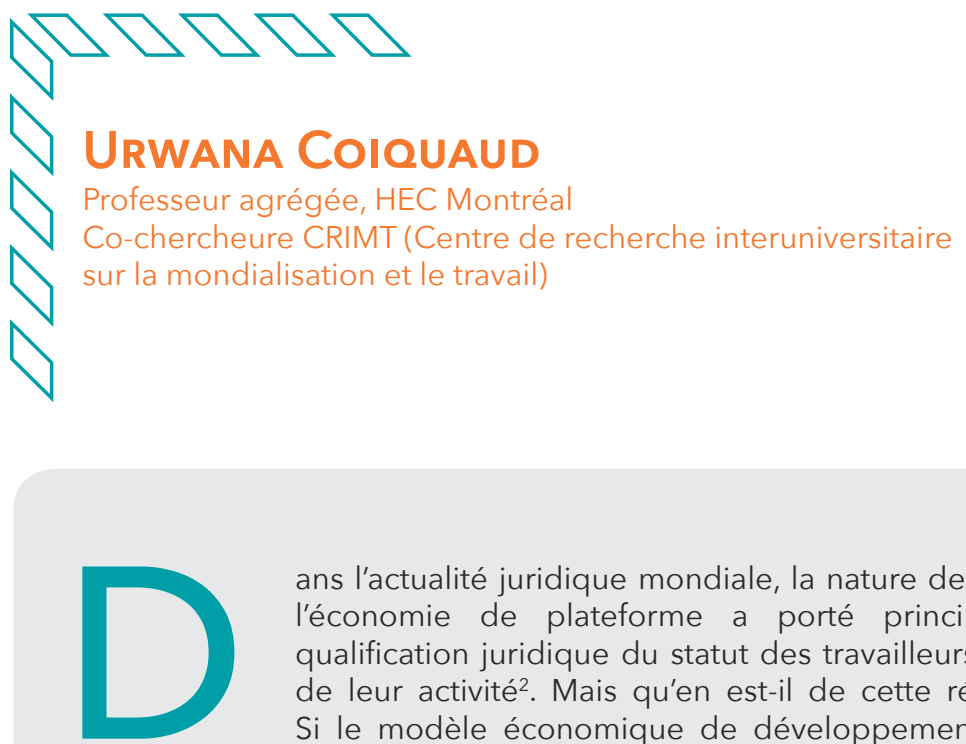

ans l'actualité juridique mondiale, la nature des litiges entourant l'économie de plateforme a porté principalement sur la qualification juridique du statut des travailleurs ${ }^{1}$ ou sur la nature de leur activité2. Mais qu'en est-il de cette réalité au Canada? Si le modèle économique de développement est réputé plus libéral que la majorité des États européens, est-ce à dire que l'avènement des plateformes dans les différents secteurs économiques se soit fait sans heurt?

Pour répondre à cette question, un simple examen des banques de données jurisprudentielles pancanadiennes révèle qu'il n'en est rien. L'effervescence de l'activité judiciaire traitant des plateformes illustre à elle seule les nombreux débats qu'elles ont suscités, voire provoqués. Au Canada, elle s'est concentrée sur la multinationale Uber qui, entre 2012 et 2014, s'est installée dans plusieurs provinces dans les secteurs du transport de personnes par taxi et de la livraison. Entre janvier 2013 et février 2019³, ce sont plus de 33 décisions qui ont été rendues à travers le pays, mais seulement deux provinces s'illustrent à cet égard : le Québec avec 22 décisions (dont un peu moins de la moitié impliquent les autorités fiscales) et l'Ontario avec 10 décisions. À elles seules, les deux provinces couvrent la quasi-totalité des litiges.

1 Par exemple : en France, Arrêt n¹737 du 28 novembre 2018 (17-20.079) - Cour de cassation - Chambre sociale- ECLI:FR:CCASS:2018:SO01737; Royaume-Uni, Uber BV v Aslam [2018] EWCA Civ 2748; USA (Californie), Dynamex Operations West, Inc. v. Superior Court of Los Angeles, n 222732 (Cal. Sup. Ct. Apr. 30, 2018).

2 Arrêt Asociacion Profesional Elite Taxi c. Uber Systems Spain SL, CJUE, 20 Déc. 2017, aff. C-434/15.

3 Données récupérées le 20 février 2019 sur le site Canlii de l'Institut canadien d'information juridique. 
Cette contribution s'intéressera donc aux situations québécoise et ontarienne ${ }^{4}$, les deux provinces les plus peuplées où s'appliquent un régime inspiré du droit civil pour la première et un régime de Common law pour la deuxième. Si l'objet des recours diffère entre ces deux provinces par leur nature, il existe une certaine similitude à l'égard du véhicule procédural employé. Dans les deux cas, l'action collective ${ }^{5}$ a tenté de contrecarrer les activités d'Uber soit pour requalifier le contrat de service en contrat de travail pour l'Ontario, soit pour réclamer des dommages résultant d'une activité professionnelle exercée au mépris des règles qui la gouvernent, pour le Québec.

Faut-il dès lors s'étonner que les tribunaux québécois n'aient pas eu à se saisir d'une demande en requalification d'un contrat de service en contrat de travail entre Uber et un particulier? Une réponse nuancée s'impose. Premièrement, l'industrie du transport de personnes par taxi dans les années 80 a mis en doute le statut des chauffeurs de taxi (étaient-ils des salariés ou des travailleurs autonomes? Et pouvaient-ils se syndiquer?). À la première question, les chauffeurs de taxi albertains furent rabroués par la Cour suprême du Canada ${ }^{6}$. Cet échec ne désarçonna pas d'autres chauffeurs de la tentative d'obtenir l'accès à la syndicalisation. En effet, un tribunal inférieur québécois sera de nouveau saisi de la question, mais repoussera cette tentative de requalification, mettant ainsi un terme à d'éventuelles velléités de requalification ${ }^{7}$. Cet état de la jurisprudence ne devrait pas

4 Le régime fédératif institué par la Constitution canadienne répartit la compétence législative par matière entre l'État fédéral et les provinces. Les conditions et les relations de travail n'étant pas visées par le texte, ce sont les tribunaux qui ont tranché la question. Il a été décidé qu'en principe, la compétence législative usuelle en cette matière revient aux juridictions provinciales, tandis que le Parlement du Canada dispose en ce domaine d'une compétence d'exception, mais qui devient exclusive dès lors qu'il s'agit de réguler les relations de travail à l'égard des entreprises pour lesquelles le Parlement est habilité à légiférer. En clair, cela signifie qu'il existe au Canada 14 régimes de relations de travail (10 provinces, 3 territoires et le régime fédéral). Uber effectuant du transport ou de la livraison locale répond de la réglementation provinciale.

5 En vertu de l'article art. 571 du Code de procédure civile, RLRQ c C-25.01 : « L'action collective est le moyen de procédure qui permet à une personne d'agir en demande, sans mandat, pour le compte de tous les membres d'un groupe dont elle fait partie et de le représenter. Outre une personne physique, une personne morale de droit privé, une société ou une association ou un autre groupement sans personnalité juridique peut être membre du groupe ».

Bien qu'il existe des différences significatives d'une juridiction à l'autre, l'action collective est souvent vue comme un instrument de justice sociale qui, sur la base d'une autorisation judiciaire, permet à une action de se poursuivre sur une base collective. L'autorisation (selon la procédure québécoise) ou la certification (en Ontario) consiste à filtrer les demandes, c'est-à-dire d'examiner le sérieux de la cause qui implique souvent de nombreux justiciables, de démontrer l'existence d'un groupe présentant un préjudice similaire ou connexe et l'existence de rapports communs avec le défendeur, et de valider le statut de "représentant adéquat » du demandeur, puisqu'il doit disposer des qualités nécessaires pour assurer une protection des droits et des membres absents. Finalement, cette procédure aura pour effet ultérieur d'accorder la force de chose jugée d'un jugement au fond envers les membres du groupe. L'action collective est donc un « véhicule procédural » qui ne modifie ni ne crée des droits substantiels (Bisaillon c. Université Concordia, [2006] 1, par. 15 RCS 666, 2006 CSC 19 (CanLII), par. 15 [le juge Lebel pour la majorité]).

6 Yellow Cab Ltd. c. Board of Industrial Relations, 1980, 2 R.C.S. 772.

7 Union des chauffeurs de taxis, Métallurgistes unis d'Amérique, local 9217 c. Taxi Cartier inc., D.T.E. 89 T937 (C.T.); M. D'Amour, « Des tentatives de s'en sortir », Vie ouvrière, 1981, 157, p. 46; U. Coiquaud, "Le difficile encadrement juridique des travailleurs autonomes en situation précaire : Le cas des chauffeurs locataires de taxi », Relations industrielles, 2009, 64, n¹, p. 98. 
pour autant décourager de nouvelles tentatives de requalification du contrat de service en contrat de travail. Nous prétendons au contraire que l'arrivée d'Uber sur le marché québécois peut offrir de nouvelles opportunités aux chauffeurs qui œuvrent dans cette industrie par son intermédiaire. Ce point ne fera pourtant pas l'objet de développement dans le présent texte. II explique cependant pourquoi l'arrivée d'Uber n'a pas suscité les débats habituels autour de la nature juridique du contrat. Les tribunaux ont plutôt été saisis par le biais d'actions collectives initiées par les chauffeurs traditionnels dans le but d'obtenir des dédommagements liés, dans un premier temps, à l'inaction du gouvernement à la suite de l'arrivée d'Uber sur le marché du transport par taxi puis, dans un deuxième temps, à la nouvelle réglementation négociée entre le gouvernement et Uber.

Pour observer les débats judiciaires entourant la requalification du contrat liant Uber à un particulier, prestataire de service, il faut se tourner vers l'Ontario. Bien que ce débat ne soit pas encore tranché au fond et qu'il ait pris, là aussi, la forme d'une action collective contre Uber, il est né d'une question préjudicielle. Cette dernière lève alors le voile sur l'organisation de l'accès à la justice des travailleurs ayant contracté avec la multinationale. Dans un premier temps, la situation québécoise sera exposée (I) pour traiter ultérieurement de celle de l'Ontario (II).

\section{I - UNE RÉGLEMENTATION TARDIVE ET ACCOMMODANTE À L'ÉGARD D'UBER AU QUÉBEC À L'ORIGINE D'UN RECOURS COLLECTIF}

Après plus de deux années (2014 à 2016) d'activité illégale de transport par taxi, un dispositif réglementaire temporaire inédit a été négocié entre la multinationale Uber et le gouvernement québécois ${ }^{8}$. Cette expérimentation réglementaire et institutionnelle appelée " projet pilote » a engendré la coexistence de deux cadres normatifs; l'un, nouveau, destiné aux chauffeurs d'Uber et l'autre ancien encadrant depuis plusieurs décennies les chauffeurs traditionnels.

Les conséquences de cette inaction n'ont pas tardé à se faire sentir. Entre 2011 et 2014, chez les chauffeurs de taxi traditionnels, neuf faillites ont été recensées. Elles sont passées à 27 entre 2015 à 2017\%. Sur l'île de Montréal, une diminution de 38,6 \% de la valeur des permis de propriétaires de taxi a été enregistrée entre janvier 2014 et juin 2017, passant respectivement de 192694 \$à $118375 \${ }^{10}$. L'avènement d'une réglementation spécifique et temporaire pour Uber dès 2016 n'a cependant pas résolu l'écart réglementaire entre les deux types de chauffeurs. Par exemple, les modalités d'entrée dans la profession ont été assouplies pour les chauffeurs d'Uber; certaines différences peuvent être appréciées dans le tableau 1.

8 Arrêté $n^{\circ}$ 2016-16 du ministre des Transports, de la Mobilité durable et de l'Électrification des transports, 30/09/2016, https://www.ctq.gouv.qc.ca/fileadmin/documents/publications/Arrete MinisterielUBER.pdf et https://www.ctq.gouv.qc.ca/taxi/projets pilotes dans le secteur du transport par taxi.html. Entente signée entre le ministère du Transport et le Directeur général d'Uber Canada inc. le 9 septembre 2016.

9 M. Payen, «Les faillites de chauffeurs de taxi ont triplé depuis Uber», TVA Nouvelles, 23 mars 2018, https://www.tvanouvelles.ca/2018/03/23/les-faillites-de-chauffeurs-de-taxi-ont-triple-depuis-uber

10 https://www.transports.gouv.qc.ca/fr/entreprises-partenaires/entreprises-taxi/Documents/ rapport-ministre.pdf 
Tableau 1 - Comparaison des exigences de formation entre les chauffeurs traditionnels et chauffeurs Uber en 2017

\begin{tabular}{|l|l|}
\hline \multicolumn{1}{|c|}{ Chauffeur de taxi traditionnel } & \multicolumn{1}{|c|}{ Chauffeur partenaire Uber } \\
$\begin{array}{l}\text { Formation (effectuée par des établissements } \\
\text { accrédités) }\end{array}$ & $\begin{array}{l}\text { Formation : 20 h avant 2017 et 35h à } \\
\text { partir de 2017 : Uber offre lui-même une } \\
\text { formation à ses « partenaires-chauffeurs » et } \\
\text { de base; } 7 \text { h h transport personne handicapée) } \\
\text { valide les connaissances acquises. }\end{array}$ \\
$\begin{array}{l}\text { Coût } 1200 \$ \\
\text { Le } 21 \text { septembre } 2017, \text { le gouvernement a adopté } \\
\text { un nouveau projet pilote qui vient réduire le } \\
\text { nombre d'heures de formation à 35 h et privatise le } \\
\text { processus, puisque les intermédiaires de services } \\
\text { ou les propriétaires de permis peuvent eux-mêmes } \\
\text { délivrer la formation et procéder à l'évaluation des } \\
\text { connaissances }{ }^{11} \text {. }\end{array}$ & \\
\hline
\end{tabular}

Il faut aussi souligner d'importantes distinctions entre les deux catégories de chauffeurs du point de vue tarifaire; les chauffeurs d'Uber ne se voient pas imposer le cadre réglementaire rigide des acteurs traditionnels de l'industrie (tableau 2).

Tableau 2 - Comparaison des tarifs entre le taxi traditionnel et Uber en 2019

\begin{tabular}{|l|l|c|c|c|c|c|}
\hline \multicolumn{2}{|c|}{} & Taxi traditionnel & \multicolumn{4}{|c|}{ Uber } \\
\hline \multirow{2}{*}{2019} & \multirow{2}{*}{ Prise en charge } & $3,50 \$$ & UberX & Uber XL & Uber Select & Taxi $^{*}$ \\
\cline { 3 - 7 } & & 1,90 & 4,30 & 4,30 & 3,45 \\
\cline { 2 - 7 } & Tarif au km & $1,75 \$$ & 0,79 & 1,33 & 1,75 & 1,70 \\
\cline { 2 - 7 } & Temps d'attente & $0,65 \$$ & 0,19 & 0,22 & 0,27 & 0,63 \\
\cline { 2 - 7 } & Course minimum & $X$ & 6,80 & 8,80 & 8,80 & 3,45 \\
\cline { 2 - 7 } & Frais de réservation & $X$ & 2,10 & 2,10 & 2,10 & $\times$ \\
\cline { 2 - 7 } & Frais annulation & $X$ & 5,00 & 5,00 & 5,00 & 5,00 \\
\hline
\end{tabular}

* Le site d'Uber consulté en janvier 2019 n'affichait pas l'augmentation des tarifs en vigueur depuis le 1er juin 2018 alors même qu'elle avait été décidée par la Commission des transports du Québec après consultation publique et avalisée dans la décision : 2018 QCCTQ 0740 https://www.ctq.gouv.qc.ca/fileadmin/documents/ Decision/20180CCTQ0740.pdf

Ainsi, Uber dispose d'une triple souplesse tarifaire : par la gamme de services offerts, par l'ajout de frais facturables au client et par la variation des prix en fonction de la demande (surging price) dont seul Uber détermine les paramètres pour configurer les algorithmes.

11 https://www.ctq.gouv.qc.ca/fileadmin/documents/secteurs/taxi/Arrete Ministeriel 20170907.pdf 
Cette mise en contexte est utile pour comprendre les actions judiciaires menées par les acteurs traditionnels à l'encontre d'Uber. Si certaines se sont soldées par un échec ${ }^{12}$, d'autres ont été couronnées de succès. C'est le cas en particulier des demandes d'autorisation d'actions collectives contre Uber ${ }^{13}$, contre la Procureure Générale du Québec (PGQ) et le gouvernement du Québec ${ }^{14}$ qui ont été accordées. Dans un premier jugement, la Cour supérieure a autorisé le demandeur à représenter toutes les personnes physiques ou morales, titulaires de permis de propriétaires de taxi ou de permis de chauffeurs de taxi depuis le 28 octobre 2014, dans le cadre d'une action collective en dommages-intérêts visant à indemniser la perte de revenus des propriétaires et des chauffeurs ainsi que la perte de valeur des permis de propriétaires de taxi ${ }^{15}$. Dans le deuxième jugement ${ }^{16}$, la Cour a autorisé l'action collective contre la PGQ et le gouvernement dans le cadre d'une action en responsabilité civile. Le demandeur estimait qu'en omettant d'appliquer la législation relative au transport par taxi à l'égard d'Uber et ses chauffeurs, il en est résulté une perte de revenus et de valeur des permis de propriétaires ou de chauffeurs de taxi dont ils se sont rendus responsables.

II restera à démontrer notamment qu'Uber et ses chauffeurs exerçaient des activités en infraction avec la réglementation, et que la PGQ et le gouvernement ont permis et toléré qu'Uber et ses chauffeurs fassent une concurrence déloyale aux acteurs traditionnels, engageant dès lors leur responsabilité civile. À ce stade, le tribunal doit déterminer s'il autorise le recours. Il filtre donc la demande au mérite de la cause, notamment en examinant si les faits allégués sont tenus pour avérés. Il s'agit donc d'une première victoire procédurale ${ }^{17}$.

Par ailleurs, en 2017, plusieurs associations de chauffeurs et de propriétaires ont cherché à obtenir l'annulation du projet pilote. Ces demandes ont été rejetées en première instance, mais ont été portées en appel ${ }^{18}$. La Cour d'appel devra donc se prononcer sur la légalité du projet pilote ${ }^{19}$ mais, en attendant, l'autorisation d'action collective contre la PGQ et le gouvernement est suspendue ${ }^{20}$.

Si le statut des chauffeurs de taxi traditionnel semble bien ancré au Québec et explique l'absence d'affaires portées par les tribunaux à l'égard des travailleurs de plateformes, il en va différemment en Ontario.

12 Regroupement des travailleurs autonomes Métallos, section locale 9840 c. Québec (Procureure générale), 2016, QCCS 4491 (Rejet de la demande d'injonction provisoire visant à suspendre I'application de l'Entente sur le Projet pilote); Regroupement des travailleurs autonomes Métallos, section locale 9840 c. Uber Technologies inc., 2016, QCCS 4626 (rejet de l'ordonnance d'injonction interlocutoire provisoire ordonnant au Ministre de suspendre la mise en application de l'Entente avec Uber Canada inc.).

13 Jean-Paul c. Uber Technologies inc., 2017, QCCS 164, par. 48.

14 Metellus c. Procureure générale du Québec, 2018, QCCS 4626.

15 Jean-Paul c. Uber Technologies inc., 2017, QCCS 164.

16 Metellus c. Procureure générale du Québec, 2018, QCCS 4626.

17 Voir supra, note 5.

18 Regroupement des travailleurs autonomes Métallos, section locale 9840 c. Uber Technologies inc., Regroupement des travailleurs autonomes Métallos, section locale 9840 c. Procureur générale du Québec, 2017, QCCS 4447 (CanLII); requête de bene esse pour permission d'appeler déférée à la formation de la Cour qui entendra l'appel, 2017, QCCA 2056.

19 ld.

20 Metellus c. Procureure générale du Québec, 2018, QCCS 4626, par. 172. 


\section{II - EN ONTARIO : PLUS QU'UN QUESTIONNEMENT AUTOUR DU STATUT DES TRAVAILLEURS DE PLATEFORME, UNE QUESTION DE JUSTICE}

Contrairement au Québec, la jurisprudence ontarienne a été saisie d'un recours collectif pour faire déclarer les chauffeurs d'Uber salariés et, à ce titre, leur permettre de bénéficier des protections et des avantages énumérés à la Loi de 2000 sur les normes d'emploi (LNE) ${ }^{21}$ dont la multinationale les aurait privés. Ce litige n'a pas été tranché au fond, puisqu'Uber a présenté une requête en suspension du recours collectif, au motif que M. Heller était lié par une clause compromissoire l'obligeant à soumettre son litige à l'arbitrage aux Pays-Bas. Le litige qui devait porter sur le statut des chauffeurs d'Uber a donc été transporté sur le terrain de l'accès à la justice de ces travailleurs. Les prochaines lignes exposeront le contenu de cette affaire qui, outre l'issue de litige favorable à M. Heller, met en lumière l'édifice contractuel, conçu par la plateforme, dans le but d'éluder ou tout au moins de tenter d'entraver l'accès à la justice de ses cocontractants.

Revenons sur les faits de cette affaire; Monsieur Heller est chauffeur-livreur d'UberEATS depuis février 2016. Il gagne entre 400 et 600 dollars par semaine pour environ 40 à 50 heures de travail et conduit son propre véhicule. Comme tous les chauffeurs d'Uber, pour avoir accès à l'application, M. Heller adhère au contrat soumis par la plateforme pour s'y connecter et aux «termes et conditions » qu'il contient. Ce contrat, long de 14 pages, est accessible sur le téléphone portable du chauffeur qui doit alors cliquer «Oui j'accepte» une première fois, puis reconfirmer son adhésion en affirmant qu'il a lu et accepté tous les contrats futurs. II appuie alors une seconde fois sur «Oui j'accepte » qui autorise ainsi le chauffeur à accéder à l'application Uber. II est à noter qu'Uber révise périodiquement l'entente, et le chauffeur doit dès lors accepter les termes de la nouvelle entente pour avoir de nouveau accès à la plateforme.

Après quelques mois de travail pour UberEATS comme chauffeur-livreur, M. Heller dépose une demande de recours collectif au nom de toute personne qui, depuis 2012, a travaillé ou continue de travailler à partir de l'une des applications d'Uber comme livreur ou chauffeur d'Uber (transport de personnes par taxi ou livreur de repas). Il estime que les chauffeurs sont plutôt des salariés d'Uber et qu'à ce titre, la multinationale aurait violé les protections et les avantages énumérés à la LNE.

Avant que le juge n'entame l'analyse au fond de la demande de M. Heller, Uber présente une requête en suspension du recours collectif. La multinationale estime que la Cour n'a pas compétence pour trancher un tel litige puisque le contrat qui unit M. Heller à Uber est un contrat commercial assorti d'une clause compromissoire attribuant la résolution du différend au tribunal d'arbitrage des Pays-Bas, conformément à la Loi sur l'arbitrage commercial international de 2017. Or l'accès à cet arbitrage impose à celui qui souhaite voir son différend résolu d'en supporter les coûts estimés, en l'occurrence à plus de 14500 dollars 22 .

21 Loi de 2000 sur les normes d'emploi, LO 2000, c 41, (ci-après citée : « LNE »).

22 Heller v. Uber Technologies Inc., 2018, ONSC 718, par. 25. 
La Cour supérieure fait droit à la demande d'Uber ${ }^{23}$. M. Heller, insatisfait, interjette appel de la décision ${ }^{24}$. Et, dans un arrêt unanime, les trois juges de la Cour d'appel renversent la décision de première instance. Ils estiment que la clause compromissoire conduit à contourner la LNE et qu'elle est inéquitable. Reprenons tour à tour ces deux motifs. En premier lieu, les juges concluent que la clause d'arbitrage conduit à soustraire les chauffeurs de la LNE. Or, cette loi s'impose à toute relation d'emploi et le droit pour un travailleur de déposer une plainte auprès du ministère de Travail constitue une norme d'emploi protégée. À l'instar de nombreuses autres contestations préliminaires, le tribunal doit seulement à ce stade déterminer si les allégations du demandeur sont susceptibles d'être prouvées. Dès lors, la question se formule ainsi : si le demandeur est qualifié de salarié, la clause d'arbitrage constitue-t-elle une façon de contourner, de se soustraire, aux normes impératives de la LNE? À cette question, les juges répondent par l'affirmative et concluent à l'invalidité de la clause d'arbitrage, car elle conduit à sous-traiter illégalement les normes d'emploi ${ }^{25}$.

En second lieu, les juges frappent la clause d'arbitrage d'invalidité, car ils la jugent inéquitable. Ils estiment qu'elle est le fruit d'une négociation injuste, qu'elle prive le demandeur de déposer plainte au ministre du Travail, comme le prévoit la LNE, que le demandeur doit, après avoir franchi plusieurs mécanismes de résolutions des conflits contrôlés par Uber ${ }^{26}$, soumettre sa plainte aux lois des Pays-Bas et présenter sa réclamation dans ce même pays en assumant des frais exorbitants. De plus, au moment de la signature, les adhérents ignorent que la juridiction choisie est celle des Pays-Bas. En outre, rien ne permet de démontrer que Heller a eu le loisir de négocier ou d'obtenir un avis juridique préalablement à la signature dudit contrat. Cet exposé des faits révèle aux yeux des juges un important déséquilibre de rapport de force entre les deux parties. Plus que cela, les juges de la Cour d'appel estiment que la clause compromissoire est le fruit d'une stratégie conçue par la multinationale dans le but de désavantager les chauffeurs vulnérables. Ils concluent que cette clause est inéquitable en vertu de la Common law. En conséquence, la clause d'arbitrage obligatoire d'Uber est déclarée invalide et les chauffeurs ou livreurs peuvent poursuivre la demande de certification du recours collectif engagé.

L'originalité du cas ontarien tient à sa capacité à démontrer que le déséquilibre du rapport de force, si caractéristique du contrat de travail, peut aussi résulter de l'absence d'accès à une justice indépendante et accessible. En l'espèce, le contrôle exercé par Uber sur ces mécanismes et les obstacles financiers qui en découlent pour le plaignant dévoilent une nouvelle facette de la façon dont certaines plateformes peuvent asseoir leur emprise

23 Heller v. Uber Technologies Inc., 2018, ONSC 718.

24 Heller v. Uber Technologies Inc., 2019, ONCA 1, (le juge Nordheimer).

25 Heller v. Uber Technologies Inc., 2019, ONCA 1, par. 23-51.

26 «Uber met à la disposition des chauffeurs un dispositif de résolution des conflits. L'application pour chauffeurs fournit un "soutien intégré", par l'entremise duquel les représentants des services à la clientèle répondent aux demandes de renseignements et aux plaintes des chauffeurs. Habituellement, les litiges impliquent de petites sommes d'argent. II y a deux niveaux de représentants du service à la clientèle. Le premier est fourni par des centres de soutien aux Philippines, le deuxième, situé à Chicago, a une formation plus spécifique d'Uber. Si les représentants ne résolvent pas la plainte du chauffeur, Uber peut faire appel à son équipe juridique. Un chauffeur peut également utiliser un centre de soutien local appelé Greenlight Hub,urwana qui fournit un soutien technique pour les applications des chauffeurs du Canada. », Heller v. Uber Technologies Inc., 2018 ONSC 718, par. 22-23. 


\section{CANADA}

sur ces «petits entrepreneurs » dépendants, en les écartant des cadres réglementaires existants et en les éloignant de la justice.

En conclusion, s'il faut admettre qu'à ce jour, le Canada n'a pas offert à la scène judiciaire une décision ou un arrêt phare capable de faire vaciller le modèle d'affaire d'Uber, l'état de la jurisprudence dans les deux provinces étudiées montre les germes d'une meilleure prise de contrôle par le droit des activités des plateformes. En effet, de tous les problèmes juridiques provoqués par Uber, deux aspects pourraient menacer frontalement son modèle d'affaires : contraindre la multinationale à respecter la réglementation sectorielle, mais aussi et surtout, requalifier ses prestataires de service en travailleurs salariés. Or, sur ces deux plans, la jurisprudence étudiée a fait des pas en avant. Les chauffeurs réclament des dommages et intérêts à la suite de pertes importantes qu'ils expliquent par le laxisme dont a fait preuve l'État face à Uber. D'autres actions cherchent à requalifier ces travailleurs en salariés. Si cette dernière action n'a pas encore été tranchée au fond, la requête formulée par Uber pour suspendre l'action collective a tourné à son désavantage en révélant les dessous des clauses d'arbitrage, imposées dans les termes et conditions du contrat auxquels adhèrent les travailleurs des plateformes. L'analyse de ces clauses et des mécanismes a révélé au grand jour le pouvoir de négociation écrasant d'Uber et la façon dont il cherchait à profiter sciemment de la vulnérabilité des travailleurs en les muselant. Ces éléments ne manqueront pas d'alimenter les débats judiciaires sur le fond le moment venu.

Pour l'heure, l'état de la jurisprudence montre que l'attitude pusillanime qui consistait à éviter de parler d'illégalité en évoquant les plateformes ou à s'aveugler volontairement est désormais révolue, et que le droit reprend ses droits. Cela est d'autant plus important que les actions judiciaires entreprises sont des actions collectives dont l'ampleur ne manquera pas de faire parler d'elles. 